Historic, Archive Document

Do not assume content reflects current scientific knowledge, policies, or practices. 



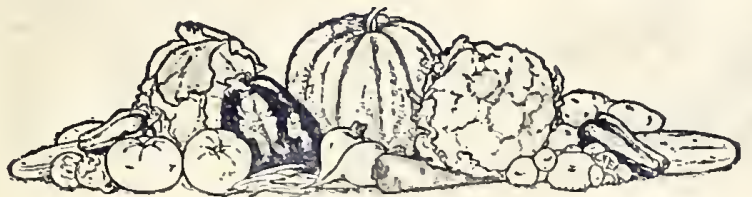

U. S. Dept. of Agriculture, Wasnirgtor.

D. C.

\section{SPECIAL PRICE LIST OF TRUE BRED SEEDS}

We know you want seeds of the best quality that can be grown and we believe that you want these as reasonably priced as possible, quality considered. We cannot secure the best seed for less money, so we are trying to reduce our selling cost for your benefit. With this in view, we are not going to the expense of getting out our usual Annual for Garden and Farm this season. Instead, we are trying to pass this saving on to our customers in reduced prices noted in this list. For those interested in descriptions and cultural directions, we still have a limited supply of our last Annual, which will be sent upon request.

In this list we have attempted to give you the low down on present market for the most dependable seeds that can be grown. Our stocks are carefully selected and tested and will please the most critical trade. If you want the most for your money in seeds, especially adapted to the South, we suggest that you place your order now and be ready for the spring planting season.

\section{DIRECTIONS FOR ORDERING}

By carefully observing the following instructions, mistakes and misunderstandings will be very largely avoided.

We pay the Postage or Express on all Vegetable Seeds and Plants in this list, except where otherwise specified. Field Seeds and Supplies are priced F. O. B. Elizabeth City; if by mail, add postage. We guarantee safe delivery of the goods at mail, add pour home.

Be sure to sign your Name, Post Office, Rural Route, and State on every order. Tell us your nearest express office, if your order is to be sent by express. Write plainly.

Ours is a cash business and our prices are niade on that basis. Customers are requested to remit when ordering. If C. O. D. shipment is desired, one-fourth of the amount of the order must accompany the order. No plants shipped C. O. D.

Buxton White Seed Company gives no warranty, express or implied, as to descripion, quality, productiveness or any other matter of any seed, bulbs or plants it sells and will not be responsible for the crop.

Prices quoted are subject to change wihout notice.

\section{PROFIT SHARING COUPONS FREE}

In filling mail orders for garden seed and supplies we include with each shipment Profit Sharing Premium Coupons, which may be used with us as cash. For every fifty cents of your order for vegetable and flower seeds taken from this list and sent us by mail you will receive a 5 cent Certificate. Suppose your first order for garden seed amounted to three dollars, pose your frst order for garden seed amounted to three dollars, you would recelve from us then later you order two dollars addional, you would receive could value of fifty in the place of cash for any goods listed to the value of fifty cents.

You may select as premiums Seeds, Bulbs, Plants Insecticides, Plant Foods, Poultry Supplies or any of the items we carry. They will be sent you absolutely free in exchange for carry. They will be sent you absolutely free in exchange for rofit Sharing Coupons. Remember all these coupons may be used as cash with us, but we will not redeem them on orders imounting to less than fifty cents. This offer applies only on garden seed and supplies ordered by mail, and is not good on field seeds and feeds. Keep our price list handy. You will want it to select the seeds and other goods which we senu you free.

\section{TRUE BRED TESTED VEGETABLE SEED}

ASPARAGUS

\begin{tabular}{llcccc} 
& \multicolumn{5}{c}{ Postpaid } \\
Washington Rustproof & Pkt. & Oz. & $1 / 4$ Lb. & Lb. \\
& $\$ 0.10$ & $\$ 0.20$ & $\$ 0.60$ & $\$ 1.80$
\end{tabular}

BEANS, GREEN PODDED BUSH

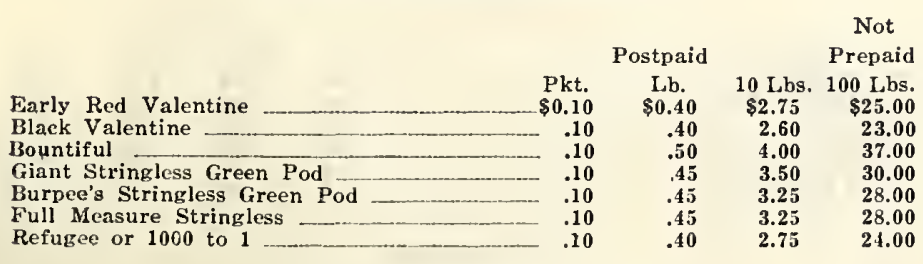

BEANS, WAX PODDED BUSH
Sure Crop Stringless Wa

$\begin{array}{rrr}.10 & .40 & 2.60 \\ .10 & .40 & 2.50\end{array}$

22.00
21.00

BEANS, POLE OR RUNNING

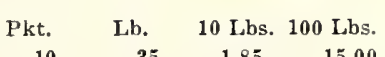

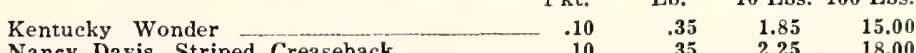

BEANS, POLE LIMA

$\begin{array}{rrrr}\text { Pkt. } & \text { Lb. } & 10 \text { Lbs. } 100 \text { Lbs. } \\ .10 & .40 & 2.25 & 18.00 \\ .10 & .40 & 2.50 & 20.00 \\ .10 & .40 & 2.60 & 22.00\end{array}$

Carolina Sieve Small Butter King of the Garden Pole

BEANS, BUSH LIMA Pkt. Lb. 10 Lbs. 100 Lbs.

Wood's Prolific Bush $\begin{array}{cccc}.10 & .40 & 2.75 & 23.00 \\ .10 & .45 & 3.25 & 28.00\end{array}$ Burpee's Improved Bush 


\section{TRUE BRED VEGETABLE SEEDS}

BROCCOLI

\begin{tabular}{cccc}
\multicolumn{5}{c}{ Postpaid } \\
Pkt. & Oz. & $1 / 4$ Lb. & Lb. \\
.10 & .40 & 1.40
\end{tabular}

Early White Cape

\section{BRUSSELS SPROUT}

Pkt. Oz. 1/4 Lb. Lb.

\section{BerTs}

Early Wonder

Croshy's Imp. Egyptian

Imp. Early Blood Turni

Crimson Globe

Swiss Chard, Spinach Beet

Chard, Spinach Beet ……_-_.___-_-_-

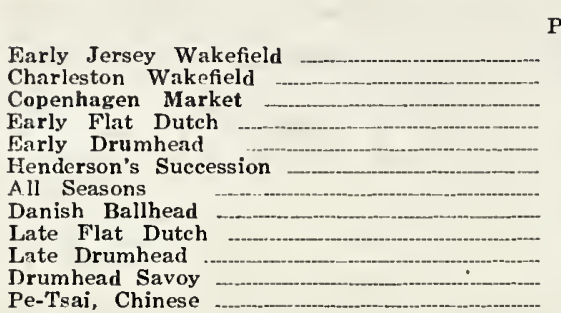

CARROTS

\begin{tabular}{rrrr} 
& & & \\
Pkt. & Oz. & 1/4 L.b. & \multicolumn{1}{c}{ I.b. } \\
.05 & .10 & .35 & $\mathbf{1 . 0 0}$ \\
.05 & .10 & .30 & .85 \\
.05 & .10 & .35 & .95 \\
.05 & .10 & .30 & .85 \\
.05 & .10 & .30 & .85 \\
.05 & .10 & .35 &. .95 \\
. .05 & .10 & .35 & $\mathbf{1 . 0 0}$ \\
.05 & .10 & .20 & .50
\end{tabular}

Danvers Half Long

Improved Long Orange

Pkt. Oz. 1/4 Lb. Lb.

Earliest Snowball

$\begin{array}{rr}\text { Oz. } & \text { 1/4 Lb. } \\ .25 & .75 \\ .25 & .75 \\ .30 & .90 \\ .25 & .70 \\ .25 & .70 \\ .25 & .70 \\ .25 & .70 \\ . .25 & .90 \\ .25 & .70 \\ . .50 & .70 \\ .30 & .80 \\ & .80\end{array}$

Lb.

2.60

2.50

2.50
2.50

3.00

2.50
2.75
2.75

Pkt. Oz. 1/4 Lb. Lb.

$\begin{array}{llll}.10 & .15 & .35 & 1.25 \\ .10 & .15 & .35 & 1.25 \\ .10 & .15 & .30 & 1.00\end{array}$

CAULIFLOWER

Pkt. Oz. T/4 Lb. Lb.

Colden Slume

Sanford's Easy Blanchin

$\begin{array}{rrrr}.10 & .25 & .75 & 2.50 \\ .10 & .60 & 2.25 & 7.50 \\ .10 & .30 & .90 & \mathbf{3 . 0 0} \\ .10 & .25 & .70 & \mathbf{2 . 2 5}\end{array}$

COLLARDS

Pkt. Oz. $\quad 1 / 4$ Lb. Lb.

North Carolina Short Stem

$\begin{array}{rrrr}\text { Pkt. } & \text { Oz. } & 1 / 4 \text { Lb. } & \text { Lb. } \\ .05 & .10 & .30 & .90 \\ .05 & .10 & .30 & .90\end{array}$

CORN, SWEET

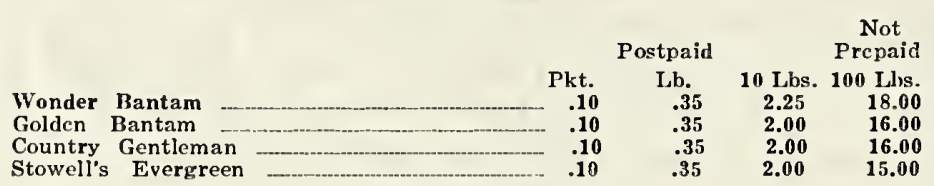

CORN, EARLY ROASTING

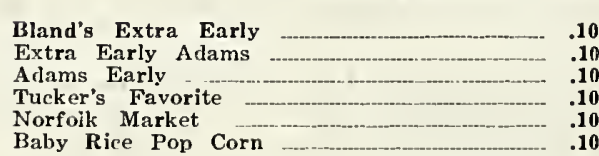

$\begin{array}{lll}.35 & 1.75 & 14.00 \\ .30 & 1.60 & 12.00 \\ .30 & 1.50 & 10.00 \\ .30 & 1.50 & 10.00 \\ .30 & 1.50 & 10.00 \\ .30 & 1.50 & 10.00\end{array}$

CUCUMBERS

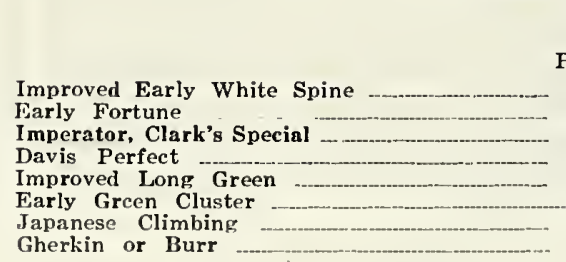

EGG PLANT

$$
\text { Postpaid }
$$$$
\begin{array}{rr}
\mathrm{Oz.} & 1 / 4 \mathrm{Lb} \\
.10 & .30 \\
.15 & .40 \\
.15 & .45 \\
.15 & .40 \\
.15 & .45 \\
.10 & .30 \\
.15 & .40 \\
.20 & .60
\end{array}
$$

1/4 Lb. Lb.

1.20

1.50
1.20
1.50

1.00

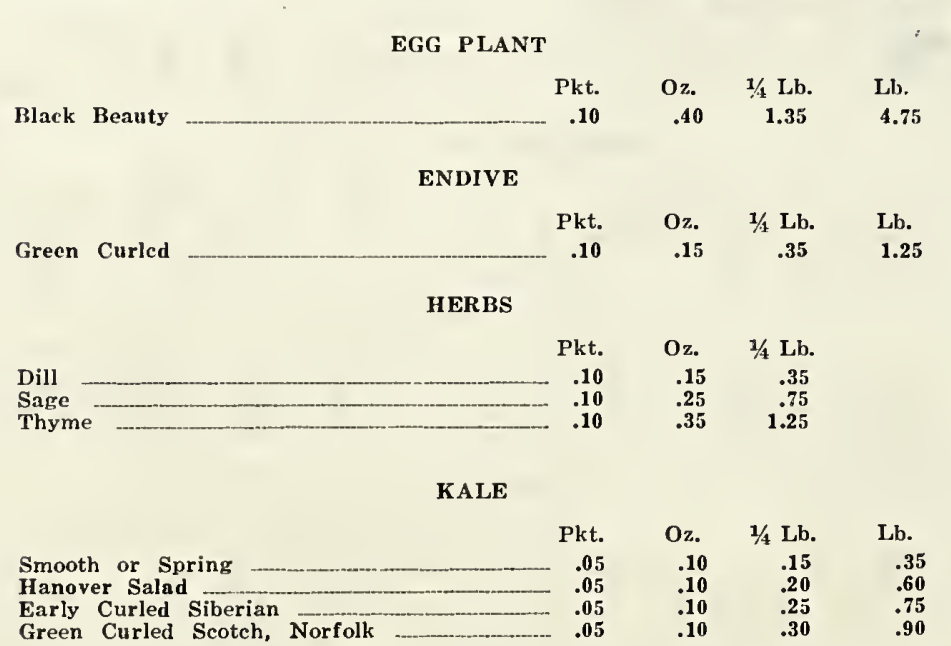

\section{LEEK}

Large American Flag

$\begin{array}{rrrr}\text { Pkt. } & \text { Oz. } & 1 / 4 \mathrm{Lb} . & \text { Lb. } \\ .10 & .20 & & \end{array}$

LETTUCE

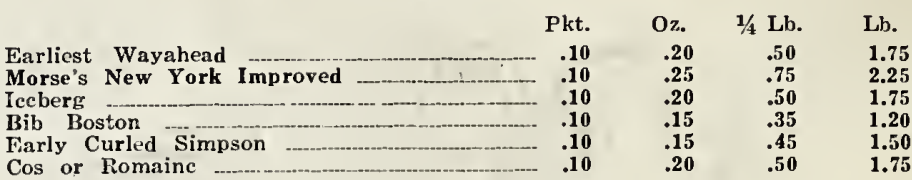

MUSTARD

Pkt. Oz. 1/4 Lb. Lb.

Southern Giant Curled

$\begin{array}{rrrr}.05 & .10 & .25 & \text { Lb. } \\ .05 & .10 & .25 & .75\end{array}$

MUSKMELON OR CANTALOUPE

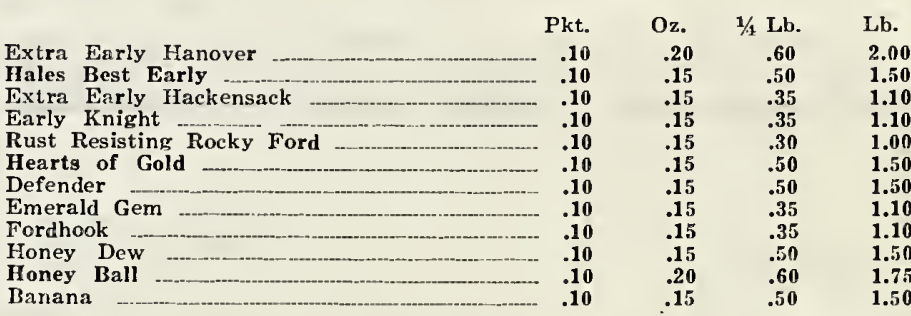

WATERMELON

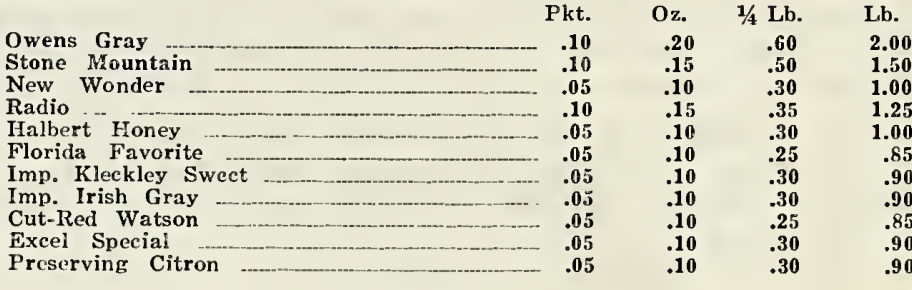

OKRA OR GUMBO \begin{tabular}{lrrrc} 
& Pkt. & Oz. & \multicolumn{1}{l}{$1 / 4 \mathrm{Lb}}$. & Lb. \\
White Velvet & .05 & $\mathbf{. 1 0}$ & $\mathbf{. 2 0}$ & $\mathbf{. 6 5}$ \\
Imp. Dwarf Green Prolific & $\mathbf{. 0 5}$ & $\mathbf{. 1 0}$ & $\mathbf{. 2 5}$ &. $\mathbf{7 0}$ \\
Perkins Mammoth Long Green & $\mathbf{. 0 5}$ & $\mathbf{. 1 0}$ & $\mathbf{. 2 0}$ & $\mathbf{. 6 5}$
\end{tabular}

ONION SEED

Extra Eariy White Pearl

Silver Skin, White Portugal

Japenese or Ebcrnezer
Prize-Taker, Spanish King

Prize-Taker, Spanish King
Yellow Globe Danvcrs
Large Red Weathersfiled

ONION SETS

$\begin{array}{rrrr}\text { Pkt. } & \text { Oz. } & \text { 1/4 Lb. } & \text { Lb. } \\ .10 & .25 & .75 & 2.75 \\ .10 & .25 & .75 & 2.75 \\ .10 & . .30 & .90 & 3.00 \\ .10 & .20 & .60 & 2.00 \\ .10 & .20 & .60 & 2.00 \\ .10 & .20 & .60 & 2.00\end{array}$

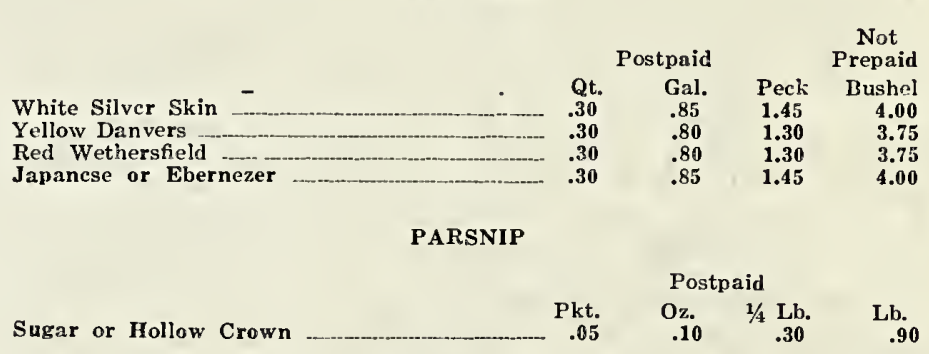

$\begin{array}{llllll}\text { Sugar or Hollow Crown } & \text { Pkt. } & \text { Oz. } & 1 / 4 & \text { Lb. } & \text { Lb. } \\ & .05 & .30 & .90\end{array}$

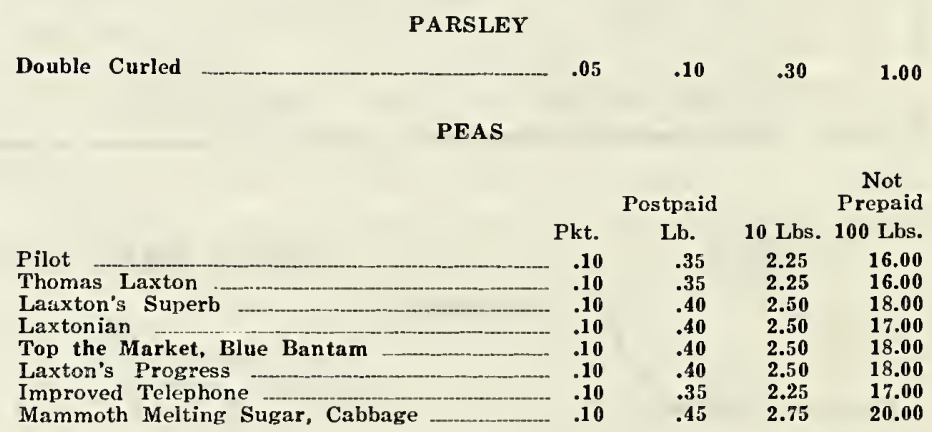

PEPPER

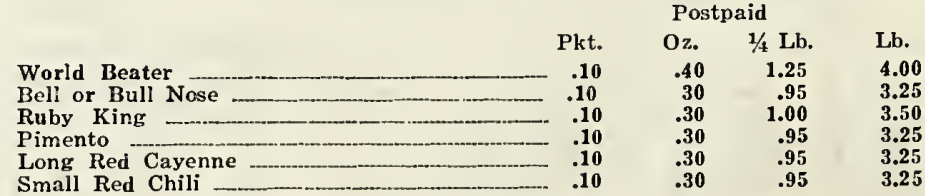

PUMPKIN

Kentucky Field

King of the Mammoths 
RADISH

\begin{tabular}{|c|c|c|c|c|}
\hline & Pkt. & Oz. & 1/1 L Lb. & Lb. \\
\hline ly Red or Scarlet Tur & .05 & .10 & .25 & .80 \\
\hline ed Scarlet Turnip & .05 & .10 & .25 & \\
\hline B Breakfast & .05 & .10 & .25 & .80 \\
\hline Iong Scarlet & .05 & .10 & .25 & .80 \\
\hline Whit & .05 & .10 & .25 & \\
\hline China Wint & .05 & .10 & .30 & \\
\hline e Chinese o & .05 & .10 & .30 & \\
\hline
\end{tabular}

RIIUIARB, PIE PLANT

Pkt. Oz. 1/ Lb. Lb.

SALSIFY, OYSTER PLANT

Mammoth Sandwich Island

SPINACH

Norfolk Savoy, Bloomsdale

SQUASH OR CYMLING

Early White Bush, Patty Pan

Giant Summer Crookn

Hubbard

Plst. Oz. 1/4 Lh. Lb.

$\begin{array}{llll}.10 & .20 & 1 / 1 & \text { Lh. Lb. }\end{array}$

$\begin{array}{rrrr}\text { Pkt. } & \text { Oz. } & 1 / \text { Lb. } & \text { Lb. } \\ .05 & .10 & .15 & .40 \\ .05 & .10 & .25 & .85\end{array}$
Pkt. Oz. 1/t Lb. Lb.

$\begin{array}{lll}.10 & .30 & .95 \\ .10 & .30 & 1.00 \\ .10 & .30 & 1.00 \\ .10 & .35 & 1.10\end{array}$

80
80
80
80
80
75
90
90

Marvana Wilt Registant

Marvelosa Wilt Resistant

Spark's Earliana

Chalk's Early Jewel

June Pink

Early Acr
Matehless

Stonc

The Brimm

Pondcrose

TOMATOES

$\begin{array}{rrrr}\text { Pkt. } & \text { O\%. } & 1 / 4 \text { Lb. } & \text { Lb. } \\ .10 & .45 & 1.25 & 4.50 \\ .10 & .40 & 1.10 & 4.00 \\ . .50 & 1.50 & 5.00 \\ .10 & .25 & .85 & 3.00 \\ .10 & .30 & 1.00 & 3.50 \\ .10 & .25 & .85 & 3.00 \\ .10 & .35 & 1.10 & 4.00 \\ -.10 & .25 & .85 & 3.00 \\ -.10 & .30 & 1.00 & 3.50 \\ .10 & .25 & .75 & 2.75 \\ .10 & .75 & 2.00 & 7.00 \\ . .10 & .50 & 1.65 & 5.75\end{array}$

TURNIP

Extra Farly White Milan

Early Red or Purple Top
Mammoth Red Top Globe

Pomeranian White Globe

Purple Top Yellow Aberdee

Golden Ball, Orange Jelly

Southern Prize

Improved Purple Top Yellow
White French, Breadstone $\begin{array}{rrrr}\text { Pkt. } & \text { Oz. } & 1 / 4 \text { Lb. } & \text { L.b. } \\ .05 & .10 & .20 & .60\end{array}$

$\begin{array}{cccc}\text { Pkt. } & 0 \% . & 1 / \text { L Lb. } & \text { Lb. } \\ .05 & .10 & .30 & 1.00 \\ .05 & . .10 & .20 & .60 \\ .05 & .10 & .20 & .60 \\ .05 & .10 & .20 & .60 \\ .05 & .10 & .20 & .60 \\ .05 & .10 & .20 & .60 \\ .05 & .10 & .20 & .60 \\ .05 & .10 & .20 & .60 \\ .05 & .10 & .20 & .65 \\ .05 & .10 & .20 & .65\end{array}$

\section{TRUE BRED VEGETABLE PLANTS AND ROOTS}

COMMERCIAL VARIETIES

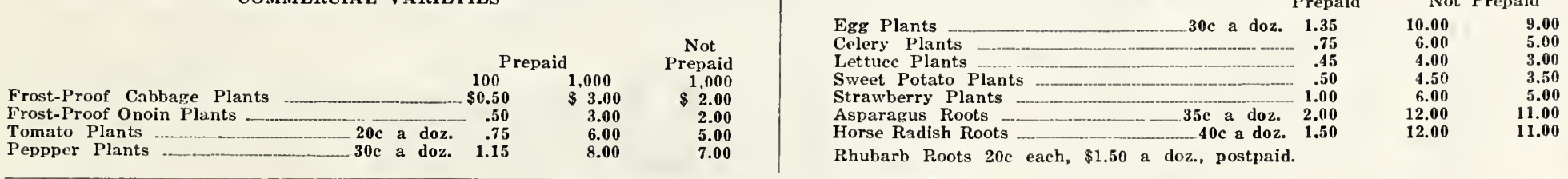

TESTED FIELD AND FARM SEED

SEED POTATOES

Current Prices at Time of Publication Subject to change Without Notice.

F. O. B. Elizabeth City

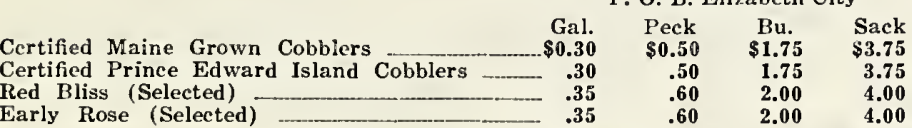
TRUE BRED COTTON SEED

F. o. B. Elizabeth City

$\begin{array}{lrrrr} & & \text { Peck } & \text { Bushel } & \begin{array}{r}5 \text {-bu. } \\ \text { per bu }\end{array} \\ \text { Mitchell's Reimproved Early King } & \mathbf{\$ 0 . 6 0} & \$ 1.85 & \$ 1.75 \\ \text { Wanamaker-Cleveland } & \mathbf{6 0} & \mathbf{1 . 8 5} & 1.75 \\ \text { Mexican Big Boll } & \mathbf{6 0} & \mathbf{1 . 8 5} & \mathbf{1 . 7 5}\end{array}$

TRUE BRED SEED CORN

\begin{tabular}{lcccr} 
& & \multicolumn{2}{c}{ Not } \\
& Prepaid & \multicolumn{2}{c}{ Prepaid } \\
Latham's Mammoth Double Eared & Qt. & Peck & Bushel \\
Currituek Horse Tooth & $\mathbf{\$ 0 . 3 0}$ & $\$ 1.00$ & $\$ 3.50$ \\
Improved Goiden Dent & $\mathbf{. 3 0}$ & $\mathbf{. 7 5}$ & $\mathbf{2 . 5 0}$ \\
\hline
\end{tabular}

TESTED CLOVER SEED

Not Prepaid

$50 \mathrm{lbs} . \quad 100 \mathrm{lbs}$.

$\$ 11.00 \quad \$ 21.00$

18.00

7.50
6.00

35.00
36.00
35.00
14.00
11.00

White Blossom Sweet Clover

TESTED GRASS SEED

Ca

Tall Meadow Oat Grass

Red Top or Herd's Gras

Italian Rye Grass
Perennial Rye Grass

Kentucky Blue Grass

Timothy Grass

SOY OR SOJA BEANS

F. o. B. Elizabeth City

Mam
Mam
Tar
Tokic
Hab
Holly
Ebo
Chi
Dix
Bilox
Lar
Mix

llybrook

Chiquit

Dixie

lixed Soys (Mammot Type)
COW PEAS

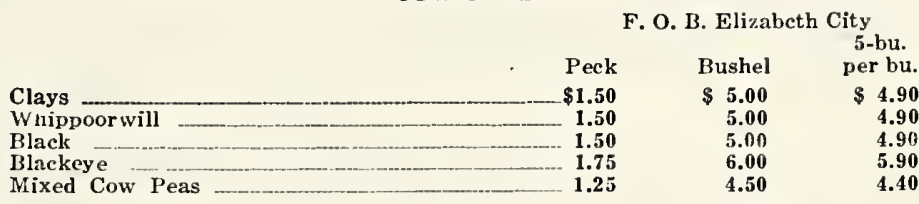

PEANUTS

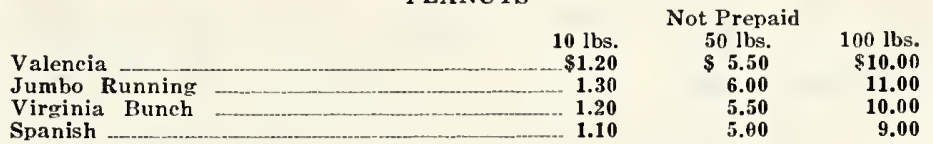

SEED OATS

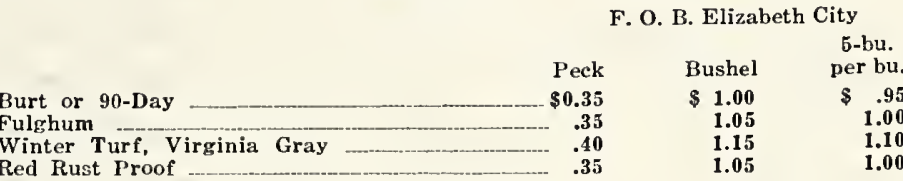

MILLET SEED

Not Prepaid

$50 \mathrm{lbs}$. $100 \mathrm{lbs}$.

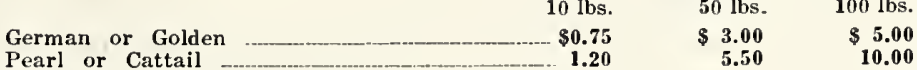

MISCELLANEOUS FIELD SEED

Not Prepaid

$10 \mathrm{lbs}$ 50 lbs. $\quad 100 \mathrm{lbs}$

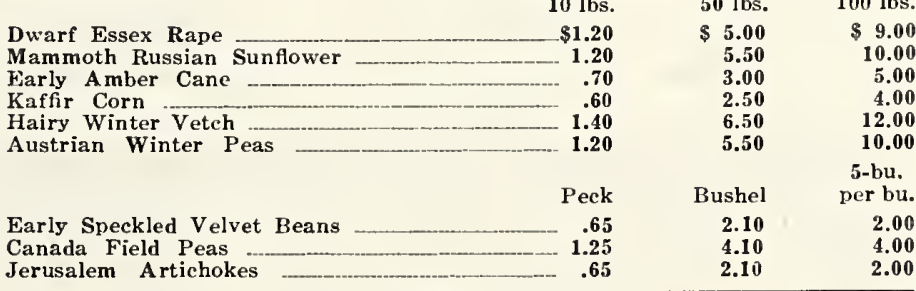

PERMANENT PASTURE

A pcrmanent pasture is one of the very best paying investments that can be made on the farm. After careful experiment, the State mixtures for this section: 8 lbs. Orehard Grass, $8 \mathrm{lbs}$. Red Top, $8 \mathrm{lbs}$. Italian Rye Grass, 8 lbs. Alsike Clover, 4 lbs Red Clover, 4 lbs. White

lover. Total seeding per acre, 40 lbs. Try it.
For poor land, where other pasture mixtures fail, the following will give heavy, succuletn green pasture: 5 lbs. Carpet Grass, 5 lbs. Dalla

EVERGREEN MIXED LAWN GRASS

We feel fully justified in stating that this is the best Lawn Grass procurable at any price. severe cold better than the various mixtures so commonly offered. Nothing but the choicest, cleanest and heavicst seeds go into it. Postpaid-Lb. 55c; 51 s. $\$ 2.35 ; 10$ lbs. $\$ 4: 50$. Not prepaid-Lb. $45 \mathrm{c} ; 5$ lbs. 


\section{INSECTICIDES, PLANT FOODS AND SUPPLIES}

Price Not Prepaid-Poisons Cannot Be Sent By Mail

BEETLE MORT-(Combined Incesticide and Fungicide.)

Lb. $45 \mathrm{c} ; 4$ lb. bag, $\$ 1.50 ; 25$ lb. case, $\$ 7.50$.

PARIS GREeN

Lb. $45 \mathrm{c} ; 2 \mathrm{lb}$ pkg., $80 \mathrm{c} ; 5$ lb. pkg., $\$ 1.90$.

ARSENATE OF LEAD

Lb. $30 \mathrm{c} ; 4 \mathrm{lb}$. bag, $\$ 1.00 ; 24$ lb. case, $\$ 5.00 ; 100 \mathrm{lbs}, \$ 18.00$.

CALCIUM ARSENATE

Lb. 20c: 4 lb. bag, 60c; 24 lb. case, $\$ 3.00 ; 100$ lbs., $\$ 9.00$.

LIME-SULPHUR DRY

Lb. $35 \mathrm{c} ; 5$ lb. can, $\$ 1.40 ; 10 \mathrm{lb}$. can, $\$ 2.40 ; 25 \mathrm{lbs}$. $\$ 4.00$.

DRY BORDO-(Bordeaux Mixture.)

Lb. $30 \mathrm{c}$; $4 \mathrm{lb}$. bag, $\$ 1.00 ; 24 \mathrm{lb}$. case, $\$ 4.80$.

POWDERED SULPHUR

Lb. $10 \mathrm{c} ; 5$ lbs., $45 \mathrm{c} ; 10$ lbs., 80c; 25 lbs. $\$ 1.75$.

SCALECIDE-(Miscible Oil.)

Quart, $75 \mathrm{c}$; gal., $\$ 1.75 ; 5$ gals., $\$ 6.25 ; 10$ gals., $\$ 10.60$.

SULFOCIDE-(Suinmer Spray.)

Pint, 70c; quart, $\$ 1.00$; gal.. $\$ 2.75 ; 5$ gals., $\$ 8.75 ; 10$ gals., $\$ 15.00$.

KAYSO-(Casein Spreader.)

$1 / 2$ lb. pkg., 30c; 2 lb. pkg., $65 \mathrm{c} ; 10$ lbs., $\$ 2.60$

BLACK LEAF 40-(Nicotine Sulphate.)

Oz. bottle, 35c; 1/2 lb., $\$ 1.25 ; 2$ lbs., $\$ 3.50 ; 10$ lbs., $\$ 11.85$

PARA-DICHLOROBENZENE-(Crystal Gas.)

Lb., 60c ; 5 lbs., $\$ 2.50$; 10 lbs., $\$ 4.75$.

PYROX-(Paste Insecticide and Fungicide.)

Lb., 50c; 5 lbs., $\$ 1.75 ; 10$ lbs., $\$ 3.00 ; 25$ lbs., $\$ 6.25$.

PLANTGARD-(Non-poisonous Insccticide.)

Small sifted top, pkg., 35c; large sifted top, pkg., 65

CHAMBERS' ANTI-SKIPPER COMPOUND-(For Curcd Meats.)

Small can, 50c; large can, $\$ 1.00$.
FIGORO MEAT PRESERVER-(Liquid Smoke.)

32 oz. jug for 500 lbs. meat, $\$ 1.50$.

CARBOLA-(Disinfecting White Paint.)

5 lb. pkg., 75c: 10 lb. pkg., $\$ 1.25 ; 50$ lbs., $\$ 5.00$.

SOILTEX-(Determines acidity of soils and proper application of lime.) Outfit complete, $\$ 1.00$.

STIMUGERM LEGUME INOCULATION-(State crop for which wanted.)

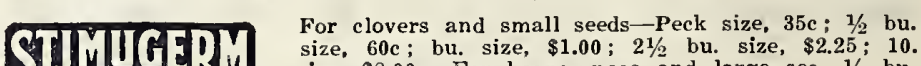

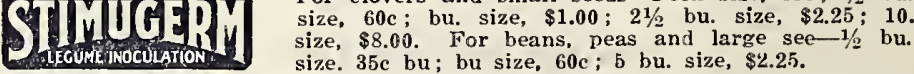

SEMESAN-(Sccd Disinfectant for General Use.)

2 oz. pkg., 50c; 8 oz. pkg., $\$ 1.60$; lb. pkg., $\$ 2.25 ; 5$ lb. pkg., $\$ 13.00$.

SEMESAN, JR.-(Sced Corn Disinfectant.)

4 oz. pkg., $60 \mathrm{c} ; \mathrm{lb}$. ukg., $\$ 1.75 ; 5$ lb. pkg., $\$ 8.00$

SEMESAN BEL-(Seed Potato Disinfectant.)

Lb. pkg., $\$ 1.75 ; 5$ lb. pkg., $\$ 8.00 ; 25$ lb. pkg.. $\$ 37.50$.

WIZARD PULVERIZED SHEEP MANURE

5 lbs., 25c; 10 lbs.., 45c; 25 lbs., $\$ 1.00 ; 50$ lbs., $\$ 1.75 ; 100$ lbs., $\$ 3.00$.

BLoOMAID-(Balanced Plant Food for Shrubs, Flowers, Vegetables.)

Tablet Form (125 tablets), 25c; Liquid Form $(8$ oz. bottle $), 50 \mathrm{c} ;$ Pul-
verised Form-lb. can, 25c; 5 lbs., 50c; 25 lbs., $\$ 1.75 ; 100$ lbs.o $\$ 5.00$.

FAIRWAY FERTILIZER-(Scientific Plant Food for Lawns and Golf Courses.) $-10 \mathrm{lb}$. bag, $\$ 1.00 ; 25$ lbs., $\$ 1.75 ; 50$ lbs., $\$ 3.00 ; 100$ lbs., $\$ 5.00$.

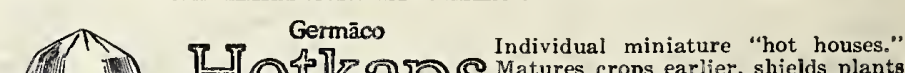
10f] $[\bigcirc[\Omega(1) S$ from frost, wind, rain and insects. New Method of A crop insurance and a money
Plant Protection makcr.

Trial pkg. of 250 with cardboard setter, $\$ 4.00$; roll of $1000, \$ 11.50 ; 5000$ at Trial pkg. of 250 with cardboard setter, $\$ 4.00 ;$
$\$ 11.00$ per thousand. Steel Hotkap Setter, $\$ 2.50$.

GATOR-HIDE MULCH PAPER-Revolutionary method of mulching plants. Eliminates weeds without cultivation, Conserves moisture, warmth, plant food, increases germination, speeds up yrowth and increases yields.

Roll of 300 yards 18 inches wide, $\$ 3.50 ; 300$ yards 36 inches wide, $\$ 7.00$

\section{POULTRY AND STOCK FEEDS}

Prices at time of publication, F, O. B. Elizabeth City. Subject to change wi thout notice.

PRATTS-AMERICA'S QUALITY FEED LINE

Pratts Buttermilk Baby Chick Food-21/2 lb. pkg., 25c; 5:3/4 lb. pkg., 50c; 10

lb. bag, 80c; 25 lbs., $\$ 1.60$; 50 lbs., $\$ 2.75 ; 100$ lbs., $\$ 5.00$.

Pratts Buttcrmilk Growing Mash

Pratts Buttcrmilk Laying Mash

$\$ 4.00$ per $100 \mathrm{lb}$. bag

Pratts Buttcrmilk Broiler Mash

Pratts Buttermilk Fattening Mash

3.75 per $100 \mathrm{lb}$. bas

4.00 per $100 \mathrm{lb}$. bag

3.75 per 100 lb. bag

3.35 per $100 \mathrm{lb}$. bas

3.60 per $100 \mathrm{lb}$. bas

3.50 per $100 \mathrm{lb}$. bag

3.00 per $100 \mathrm{lb}$. bag

3.35 per $100 \mathrm{lb}$. ba

4.00 per $100 \mathrm{lb}$. bag

3.15 per $100 \mathrm{lb}$. bag

5.00 per $100 \mathrm{lb}$. bag

3.50 per $100 \mathrm{lb}$. ba

3.25 per $100 \mathrm{lb}$. bag

3.50 per $100 \mathrm{lb}$. bag

Gubernut Laying Scratch Feed

Quaker Rolled Oats or Oatmeal

Granulated Charcoal

Crushed Oyster Shel

Pearl Poultry Grit

Choice Chicken Wheat

White Feed Corn

Choice White Fced Oats 3.75 per $90 \mathrm{lb}$. ba 1.75 per $50 \mathrm{lb}$. bag 1.25 per $100 \mathrm{lb}$. bag 3.00 per $100 \mathrm{lb}$. bag 2.25 per 2 -bu. bag 3.75 per 5-bu. bag
Cracked Corn or Hominy

2.25 per $100 \mathrm{lb}$. bag

Ground Oats 2.75 per $100 \mathrm{lb}$. bag

White Corn Mcal 2.45 per $100 \mathrm{lb}$. bag

Soft Winter Wheat Middlings 2.60 per $100 \mathrm{lb}$. bag Standard Wheat Middlings 2.50 per $100 \mathrm{lb}$. bag Pure Winter Wheat Bran 2.50 per $100 \mathrm{lb}$. bag Fine Grcen Alfalfa Meal

Feeding Fish Meal

Meat Scrap

Soy Bcan Meal 3.00 per $100 \mathrm{lb}$, bag 4.25 per $100 \mathrm{lb}$. bag

Cotton Seed Meal 4.75 per $100 \mathrm{lb}$. bag

Cotton Seed Hulls

Gray Beet Pulp

Larro Dairy Ration

Milk-Flo Dairy Feed

Molasses Horse \& Mule Fced 3.00 per $100 \mathrm{lb}$, bag 2.50 per $100 \mathrm{lb}$. bag .85 per $160 \mathrm{lb}$. bag 3.25 per $100 \mathrm{lb}$. bag 3.25 per $100 \mathrm{lb}$. bag 2.85 per $100 \mathrm{lb}$. bag Lump Rock Salt 2.50 per $100 \mathrm{lb}$. bag Morton Block Salt 1.75 per $100 \mathrm{lb}$. bag Peanut Meal .75 per $50 \mathrm{lb}$ block 1.00 per $100 \mathrm{lb}$. bag
Semi-Solid Buttermilk-5-gal, pail, $\$ 4.00$; $1 / 4$ bы. 6c per lb.; $1 / 2$ bы., 51/2c per lb.: bbl., 5c per lb.

Scmi-Solid Buttermilk with $4 \%$ Cod Liver 0il-5-gal. pail, $\$ 4.50 ; 1 / 4$ bbl., 7c per lb.

We carry a complcte line of Pratt's, Hess, Conkcy's, Burrell-Dugger Poultry and Stock Remedics, Poultry Fountains, Fceders, Incubators, Brooders and Supplies.

\section{Buxton White Seed Co.}

